\title{
Aprendizaje-Servicio y Mujeres de cine: el Making of en innovación docente
}

\section{Service-Learning and Woman in Film: the Making of teaching innovation}

\author{
Azahara Jiménez-Millán ${ }^{1}$, María García-Cano Torrico² \& Eva F. Hinojosa \\ Pareja $^{3}$
}

Fecha de recepción: 19/06/2020; Fecha de revisión: 25/07/2020; Fecha de aceptación: 07/09/2020

Cómo citar este artículo:

Jiménez-Millán, A., García-Cano, M. \& Hinojosa-Pareja, E. (2020). Aprendizaje-Servicio y Mujeres de cine: el Making of en innovación. Revista de Innovación y Buenas Prácticas Docentes, 9(2), 107-122.

\section{Resumen:}

El artículo se enmarca en un proyecto de innovación docente (2019-3-4005) desarrollado en el curso académico 2019/2020 en 2.ำ curso en los Grados de Educación Infantil y Educación Primaria en la asignatura Convivencia en la Escuela y Cultura de Paz. Se propuso utilizar el cine como texto cultural para el análisis y crítica de los sesgos de género y de los modelos patriarcales en las sociedades actuales; del mismo modo, como vehículo de acción transformadora en los espacios educativos. Mediante la metodología de Aprendizaje-Servicio (ApS) los y las estudiantes utilizaron el cine para analizar los sesgos de género y para hacer propuestas de acción socioeducativa, relacionando de esta manera aprendizajes académicos con experiencias más prácticas y vivenciales que se desarrollan fuera de las aulas. Las actividades realizadas han consistido en: selección de la temática, documentación, realización de entrevistas con expertas en materia de cine y educación, visualización de documental relacionado con la desigualdad de género, análisis reflexivo de los datos producidos y, por último, elaboración de un servicio para movilizar recursos y aprendizajes que propongan acciones alternativas a los modelos patriarcales en distintos espacios, formales y no formales. Además, el trabajo de visualización y diálogo con mujeres directoras de trabajos fílmicos ha permitido la visibilización de éstas y la interacción dentro del aula sobre la acción coeducativa.

Palabras clave: Innovación docente, Aprendizaje-Servicio, Cine, Mujeres, Coeducación.

\footnotetext{
1 Universidad de Córdoba (España), m32jimia@uco.es; CÓDIGO ORCID: https://orcid.org/0000-0002-66674636

2 Universidad de Córdoba (España), maria.garciacano@uco.es; CÓDIGO ORCID: https://orcid.org/00000002-0988-7280

3 Universidad de Córdoba (España), ehinojosa@uco.es; CÓDIGO ORCID: https://orcid.org/0000-00021677-9448
} 


\section{Abstract:}

This article is part of a teaching innovation project (2019-3-4005) developed during the 2019/2020 academic year in the 2nd year of the Infant and Primary Education Grades in the subject Coexistence in the School and Culture of Peace. It was proposed to use film as a cultural text for the analysis and criticism of gender biases and patriarchal models in today's societies; likewise, as a vehicle for transformative action in educational spaces. Through the Service-Learning (SL) methodology, students used film to analyze gender biases and to make proposals for social and educational action, thus relating academic learning to more practical and experiential experiences that take place outside the classroom. The activities carried out consisted of: selection of the theme, documentation, interviews with experts in the field of cinema and education, viewing of documentaries related to gender inequality, reflective analysis of the data produced and, finally, development of a service to mobilize resources and learning to propose alternative actions to patriarchal models in different spaces, formal and non-formal. In addition, the work of visualization and dialogue with women directors of film works has allowed the visibility of these and the interaction within the classroom on the co-educational action.

Key Words: Teaching innovation, service-learning, cinema, women, coeducation. 


\section{INTRODUCCIÓN}

La formación inicial del profesorado para la justicia social aborda explícitamente cuestiones de injusticia y desigualdad estructural que evidencian los mecanismos sociales, culturales e históricos que sitúan a colectivos en posiciones de discriminación y exclusión social vinculadas al racismo, el sexismo, el clasismo y la interacción entre ellos (Torres, 2017). Capacitar al profesorado en la etapa de formación inicial para su análisis y propuesta de acción requiere formación teórica, actitudinal y la adquisición de estrategias creativas para su transformación a favor de la equidad (Zeichner y Flessner, 2010) y la coeducación (Heredero de Pedro, 2019). El proyecto de innovación docente ${ }^{3}$ que se presenta se ha desarrollado desde esta perspectiva y con esta finalidad durante el curso 2019/2020 en los Grados de Educación Infantil y Educación Primaria de la Universidad de Córdoba y en las asignaturas Convivencia en la Escuela y Cultura de $P a z$ de ambos grados. En este contexto, su aplicación se hace especialmente propicia, ya que sus contenidos abordan temáticas de carácter socioeducativo desde la perspectiva de la Pedagogía Crítica. Desde el punto de vista didáctico y orientado a la innovación docente, en este proyecto se ha trabajado con el cine como recurso educativo e instrumento formativo para analizar, pensar y transformar los procesos de injusticia y desigualdad que experimentan las mujeres debido a los sesgos sexistas y patriarcales (Heredero de Pedro, 2019).

En particular, desde el enfoque feminista, tal como plantea la teoría fílmica feminista, se parte de la premisa de que el cine provoca un imaginario sobre las relaciones entre varones y mujeres que es productor y reproductor de arquetipos sociales, según los cuales se sitúa a las mujeres en posiciones de desigualdad en los distintos espacios y esferas del desarrollo vital (relaciones amorosas, roles sociales asignados, patrones estéticos, figuras de heroicidad y victimista, acceso a la educación, etc.) (Cruzado, 2009; Perales, 2011). El papel protagonista y decisorio que ha tenido el hombre en la industria cinematográfica asociado a la dirección, frente a la posición femenina vinculada a la interpretación u otras actividades como peluquería, maquillaje - vestuario, así como, el menor acceso de ellas al trabajo de dirección o su representación sexualizada en la gran pantalla son factores que contribuyen a la reproducción de modelos tradicionales que emulan otros ámbitos sociales y otras simbologías culturales y evidencian su desigualdad (Núñez, 2010).

Desde la innovación docente, el uso del cine brinda(r) maneras de trasladar a las situaciones cotidianas de aula la crítica social y pedagógica (Soler, Quintanilla y Aguilar, 2018, p.110) permitiendo la exposición de propuestas didácticas con ese fin. Según González-Párraga (2019), el cine como instrumento educativo ayuda a los y las docentes para alcanzar dos objetivos fundamentales: en primer lugar, para transmitir y complementar contenidos de la(s) asignatura(s) de forma atrayente y; en segundo lugar, para potenciar la cultura cinematográfica en el alumnado. En este proyecto, además, el cine se ha utilizado como elemento reflexivo (Martínez-Salanova, 2003) que permite reencuadrar la propia mirada (González-Jiménez, 2012), con el objetivo de servir de instrumento para la elaboración de propuestas alternativas y la construcción de

\footnotetext{
3 Proyecto ref. 2019-3-4005 del Plan de Innovación Docente y Buenas Prácticas Universidad de Córdoba. Disponible en: http://www.uco.es/organizacion/calidad/innovacioneducativa/es/plan-de-innovacion-envigor. El proyecto aprobado y aquí analizado fue titulado: Mujeres de cine. El cine en el Aprendizaje Servicio para la coeducación y la transformación social coordinado por María García-Cano y Eva F. Hinojosa-Pareja del Departamento de Educación.
} 
aprendizajes críticos a los patrones que reproducen los sesgos de género y sitúan a las mujeres en posiciones de desigualdad. Para ello, nos sirven de guía experiencias docentes previas exitosas (por ejemplo, Alfaya, Bueno y Navarro, 2012) que, mediante el cine, su visionado y posterior debate orientado, sirvió para analizar con el alumnado los mensajes transmitidos y comprender desde un papel crítico los roles y estereotipos asignados a las mujeres.

Para desarrollar este objetivo, se ha escogido el desarrollo de proyectos de Aprendizaje Servicio (en adelante ApS) como metodología de trabajo en el proceso de enseñanza-aprendizaje. Entre la abultada lista de definiciones de este método destaca: una propuesta educativa que combina procesos de aprendizaje y de servicio a la comunidad en un solo proyecto bien articulado, en el que los [y las] participantes se forman al trabajar sobre necesidades reales del entorno con el objetivo de mejorarlo (Puig y Palos, 2006, p.60). Aunque las primeras experiencias de ApS surgen en la década de los años 20 del siglo pasado, en la actualidad continúan siendo consideradas propuestas innovadoras cuyos principios se fundamentan en la pedagogía experiencial y reflexiva (Naranjo, 2020). Los proyectos de ApS son una apuesta pedagógica de naturaleza crítica, desde y para la transformación social, que favorece procesos participativos auténticos y relaciona los aprendizajes académicos con otros aprendizajes más prácticos y experienciales construidos desde el desarrollo de un servicio a la comunidad (Chiva, Gil, Corbatón y Capella, 2016). Como indica Deeley (2016), al adoptar una perspectiva crítica en torno al ApS, el pensamiento propio emerge como consecuencia más directa con la acción y no sólo dependiendo de la mera interacción con el profesorado o con el currículum; de esta manera, esta metodología facilita la conciencia y el pensamiento de estudiantes y motiva la acción crítica.

Coincidimos con Almacellas (2019) cuando indica que el cine es un cauce para alcanzar competencias sociales, cívicas y culturales. Elemento que vincula la metodología de ApS con el uso del cine como herramienta pedagógica. Porque más allá del uso del cine como elemento distractor-motivador en los escenarios de educación formales y no formales, existen trabajos que evidencian su valor educativo en etapas de la enseñanza obligatoria para el abordaje de temáticas de carácter social (Fueyo y Fernández, 2012; Jorge, Antolínez y Cordero, 2017) o como vehículo para la transmisión de valores (Bonilla, 2008). Especialmente relevantes son los trabajos realizados desde las teorías feministas, en los que el cine y su lenguaje de imágenes y tramas, son utilizadas como espacio privilegiado para la deconstrucción de mensajes culturales (Morales, 2015). A través del análisis de la producción cinematográfica, tanto en el ámbito nacional e internacional, se evidencia la invisibilidad de las mujeres como productoras de mensajes audiovisuales (Aguilar, 2017), así como las barreras que experimentan en relación con su liderazgo (Núñez, 2010), pero también los sesgos de género existentes cuando se representan distintos modelos sociales.

La enseñanza universitaria innovadora aúna la implementación de metodologías novedosas, experiencias piloto y proyectos que obligan a la generación de espacios de reflexión y diálogo orientados a la mejora de la practica educativa, la participación del alumnado en el proceso de enseñanza-aprendizaje y la generación de respuestas alternativas a los problemas del mundo social y educativo (Margalet y Canabal, 2013). En este proyecto, la metodología de ApS y el análisis del cine desde una perspectiva de género sirven de estrategias educativas privilegiadas tanto para visibilizar, como para deconstruir mensajes de injusticia y desigualdad y para formular propuestas transformadoras vinculadas a la equidad y la coeducación en la formación del profesorado desde las aulas universitarias. 


\section{DESARROLLO DE LA EXPERIENCIA DE INNOVACIÓN}

Tomando como referencia el posicionamiento de partida expuesto, el proyecto desarrollado se ha planteado de manera conjunta para la asignatura común de los Grados de Educación Infantil y Educación Primaria denominada Convivencia en la escuela y cultura de Paz, asignatura de carácter Básico de $2^{\circ}$ curso a un total 271 estudiantes.

En términos generales, el diseño del proyecto de innovación ha seguido los pasos típicos de la metodología en la que se enmarca de ApS, cuyo eje central consiste en la fundamentación, reflexión teórica, diseño de un servicio a la comunidad, implementación y evaluación de su impacto. Concretamente, se inició con una presentación al estudiantado de distintas temáticas vinculadas al temario de la asignatura entre los que se encontraba el cine como recurso educativo para la coeducación y la transformación social. A continuación, fue seleccionada dicha temática por parte de 1 equipo (constituido por 4-5 estudiantes) en cada clase de las 4 en las que se ha ofertado. Posteriormente, el alumnado procedió a la búsqueda y lectura de bibliografía sobre cine y género; indagación y realización del trabajo de campo, mediante entrevistas con expertas ${ }^{4}$ en cine y género, análisis de documentación y material fílmico. La discusión posterior entre el material obtenido en el campo y la literatura les proporcionó claves para el diseño, implementación y evaluación de un servicio que propusieron para ser llevado a cabo en distintos escenarios y con distintas audiencias. Finalmente, procedieron a la exposición pública del trabajo realizado en equipo en un evento organizado por las profesoras coordinadoras y partícipes del proyecto en las zonas comunes de la Facultad de Educación y que se denominó Feria de la Convivencia.

\subsection{Objetivos y competencias}

Dado el carácter socioeducativo y reflexivo de los títulos de Ciencias Sociales en general y de los grados de Educación en particular, se diseña un proyecto que sigue la metodología ApS con los siguientes objetivos:

1. Promover la indagación autónoma por parte del alumnado explorando recursos que le permitan conocer sobre: cine dirigido por mujeres, educación y género.

2. Explorar en los conocimientos, experiencias, saberes y prácticas de agentes sociales sobre cine dirigido por mujeres, educación y género a través de estrategias de investigación social como la entrevista, la observación y el análisis documental.

3. Visualizar y analizar películas dirigidas por mujeres para explorar los potenciales educativos.

4. Conocer y experimentar, mediante talleres, proyectos y experiencias concretas sobre el uso del cine como instrumento para coeducación y la transformación social.

5. Diseñar un servicio a la comunidad de carácter educativo relacionado con el cine dirigido por mujeres para el espacio educativo.

6. Realizar el servicio a la comunidad diseñado en un contexto educativo (escuela, Facultad, contexto de educación no formal).

7. Elaborar un informe del proyecto de Aprendizaje-Servicio sobre el cine dirigido por mujeres como estrategia para la coeducación y la transformación social.

\footnotetext{
${ }^{4}$ Agradecemos la disponibilidad y participación para el desarrollo de las entrevistas de Marina FuentesGuerra, Araceli Velasco y Consuelo Borreguero, miembros de la Comisión Cine de Mujeres de la Cátedra de Estudios de las Mujeres Leonor de Guzmán de la Universidad de Córdoba. http://www.uco.com.es/catedrasyaulas/catedramujeres/introduccion.php
} 
8. Evaluar, analizar y difundir el impacto del proyecto de innovación en el aprendizaje y en la satisfacción del alumnado participante.

Las competencias que han sido trabajadas vinculadas a los objetivos del proyecto para el Grado de Educación Infantil (GEI) y Educación Primaria (GEP) están relacionadas con el trabajo cooperativo y la interacción entre pares, así como el desarrollo de actitudes y habilidades para el desarrollo de actividades colectivas (CM1.6 [GEI] y CM2.7 [GEP]); el análisis crítico de temas relevantes de la sociedad y aspectos relacionados con género y discriminación e inclusión social (CM3.4 [GEl]) para la construcción de una ciudadanía activa y democrática (CM2.8 [GEP]).

\subsection{Metodología}

La metodología empleada en este proyecto ha seguido el proceso de ApS lo que ha implicado un proceso de acciones presididas por la acción, la indagación, el diseño de actuaciones (servicio) y la evaluación por parte del alumnado, dirigido y orientado por las profesoras de las asignaturas. De forma detallada y con un sentido procesual, las actividades llevadas a cabo, así como los recursos utilizados en cada caso se muestran en la Figura 1.

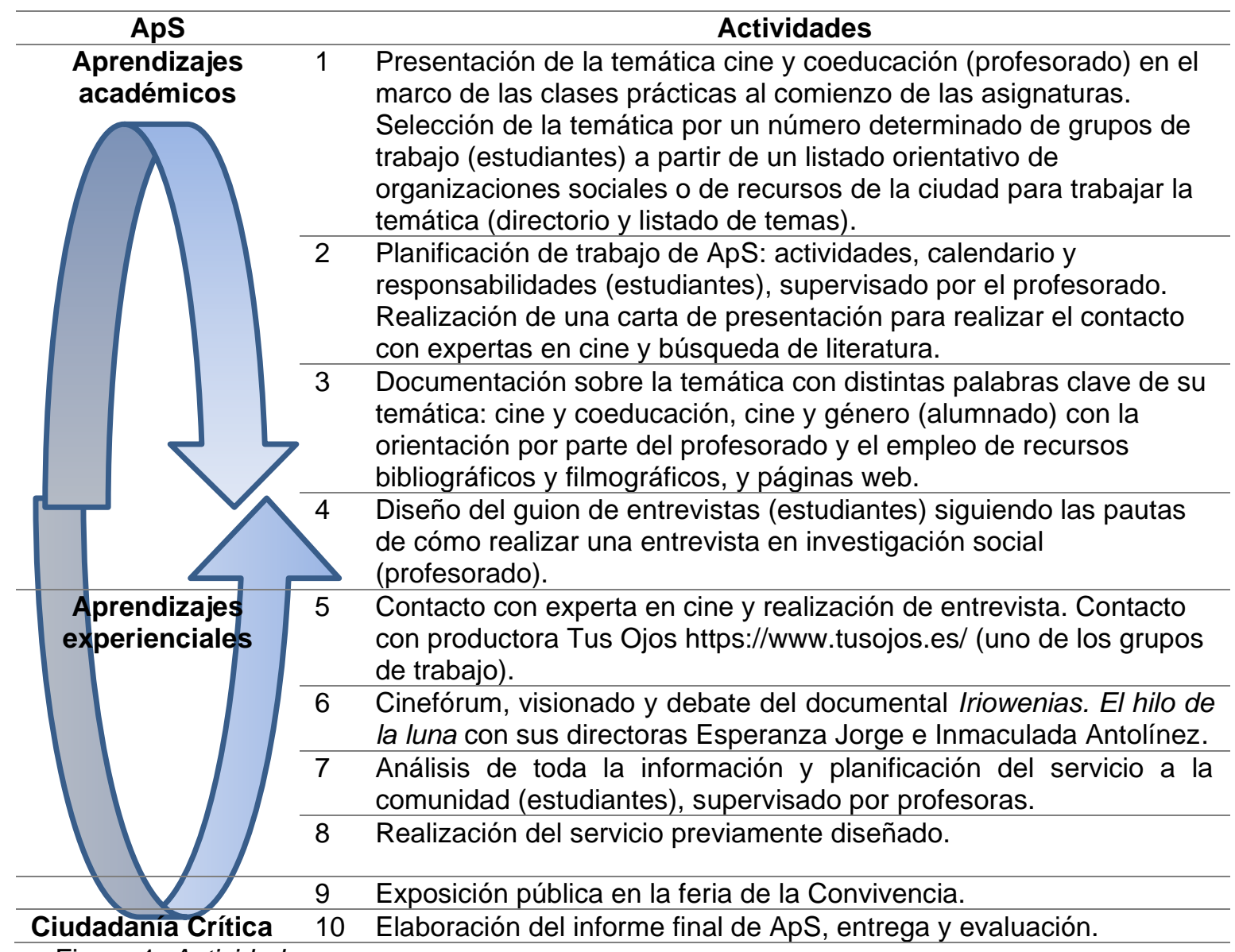

Figura 1. Actividades

Fuente: Elaboración propia

\subsection{Evaluación del Proyecto}

La evaluación del proyecto se ha llevado a cabo en diferentes momentos, ha tenido distintas finalidades y estrategias. De forma más concreta: 
Evaluación del alumnado. El principal instrumento de evaluación ha sido el Informe final de ApS realizado por el alumnado sobre cine y coeducación, orientado y calificado por las profesoras de la asignatura. El objetivo perseguido ha sido valorar su aprendizajes y adquisición de competencias a partir de la calidad y compresión que demuestra sobre la materia. El informe elaborado por el profesorado ha seguido la estructura del proceso seguido, poniendo especial atención en la conexión entre el trabajo de indagación realizado, el proceso de indagación documental y realizando las entrevistas o a través del material analizado y la experiencia de diseño e implementación del servicio realizado.

Evaluación del proyecto de innovación. De forma inicial preguntamos al alumnado a través de un cuestionario elaborado ad hoc algunos aspectos que sirvieron para evaluar la opinión sobre cine y educación desde la perspectiva del alumnado. Algunas respuestas, sirvieron para valorar el interés y oportunidad del trabajo en esta materia. Por ejemplo, en una escala de 1 a 5 (nada-mucho) el 51\% reconocieron necesitar mucha formación sobre cine viendo oportuno utilizarla como herramienta educativa (Figura 2).

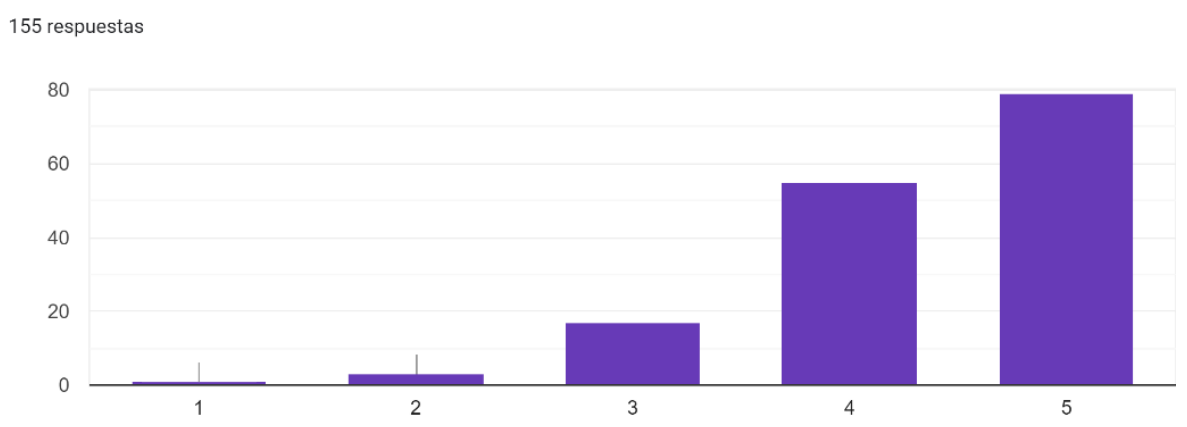

Figura 2. Resultados del cuestionario de evaluación: Como futuro educador o educadora necesito más formación sobre cómo utilizar el cine como herramienta educativa

Además, después de la actividad de formación realizada, reconocen que el cine es una herramienta adecuada para trabajar cuestiones relacionadas con el análisis de la desigualdad de género (Figura 3) y hacer propuestas para la transformación social (Figura 4).

155 respuestas

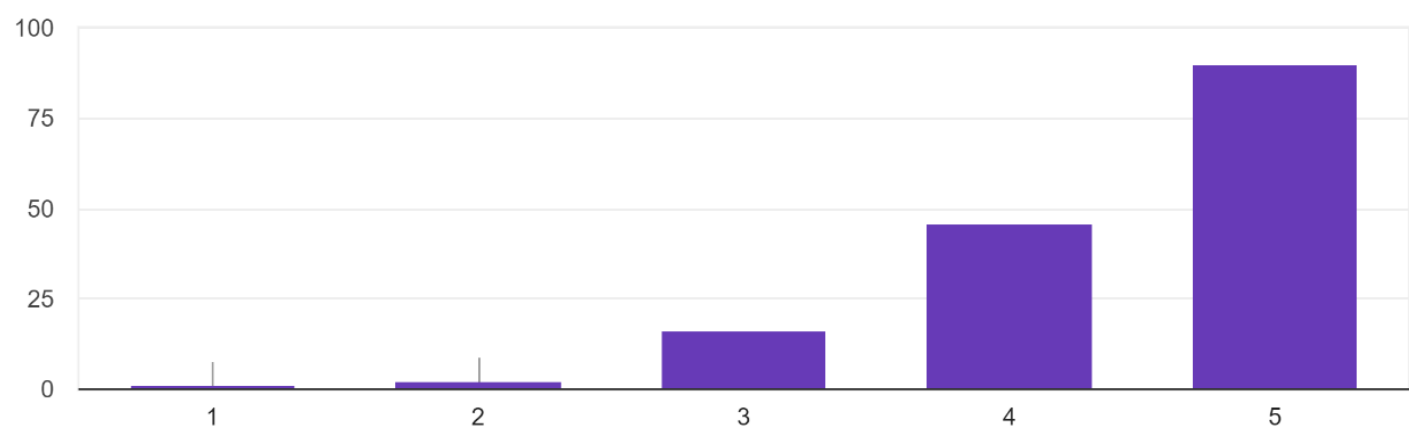

Figura 3. Resultados del cuestionario de evaluación: El cine es una herramienta de utilidad para reflexionar sobre la desigualdad de género en la sociedad. 
155 respuestas

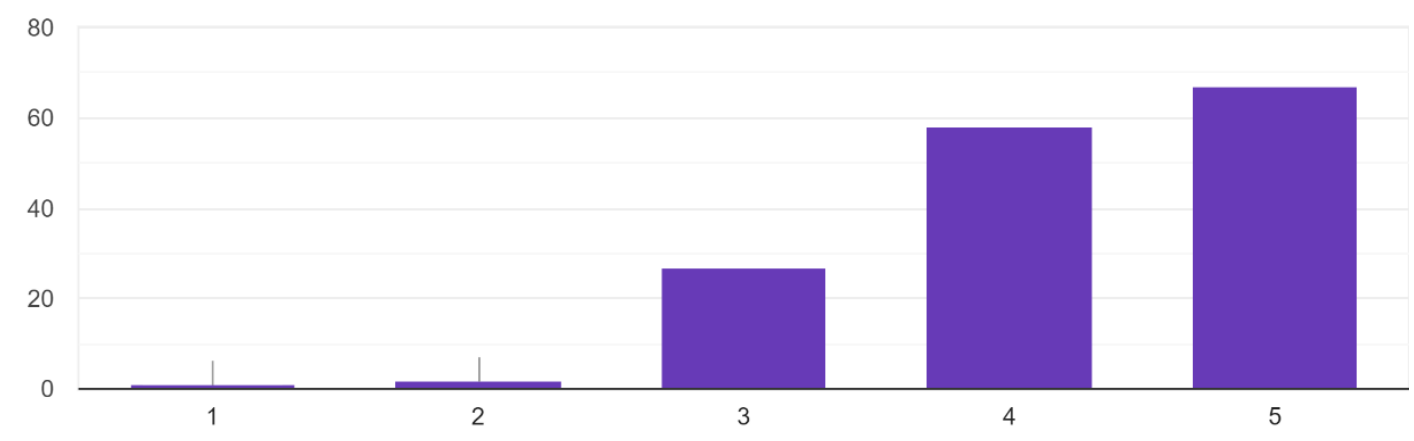

Figura 4. Resultados del cuestionario de evaluación: El cine es un instrumento para la transformación de los roles de género en la medida que me permite imaginar formas alternativas.

El nivel de satisfacción del alumnado en la actividad de visionado y debate del del documental Iriowenias. El hilo de la luna (actividad 6) se valoró mediante cuestionario y fue contestado por el alumnado asistente al finalizar dicha actividad. A tenor de los resultados obtenidos, se considera un alto grado de logro en los objetivos planteados, dado que muestran un $86,8 \%$ de grado de satisfacción con la actividad realizada (en una escala Likert de 5 puntos, 58,1\% participantes otorgan la puntuación máxima y $29,7 \%$ indican 4). Asimismo, la mayor parte del alumnado cuando asiste a la actividad $(80,6 \%)$ manifiesta que el cine puede ser una herramienta para la transformación de roles de género $(43,2 \%$ señalan la puntuación máxima y $37,4 \%$ indican 4 sobre 5).

\section{RESULTADOS}

De manera general, los resultados alcanzados con el desarrollo de la experiencia de innovación docente descrita han sido muy satisfactorios en relación con los objetivos propuestos. Como se ejemplificaba en la Tabla 1, constituye un proceso de ida y vuelta, es decir, entre el conocimiento académico, las experiencias y los procesos de reflexión y acción. Por ello, aunque los aprendizajes constituyen un todo sin límites claros, entre unos y otros, a continuación, se presentan los resultados obtenidos con relación a los aprendizajes del alumnado estructurados en tres líneas distintas: aprendizajes académicos a través de la indagación teórica, aprendizajes experienciales y, finalmente, aprendizajes vinculados a la adquisición de competencias de ciudadanía social y crítica.

\subsection{Aprendizajes académicos}

De manera general, la temática propuesta por el equipo docente -cine de mujeres- ha sido de interés por parte del alumnado, siendo seleccionado en todos los grupos-clase donde fue presentado, como eje sobre el que realizar su proyecto de ApS. La calidad de los trabajos ha sido notable recibiendo una calificación por encima de 7 puntos en todos los casos (objetivo 7).

La indagación teórica autónoma por parte del alumnado (objetivo 1) les ha permitido conocer cine dirigido por mujeres y vincularlo a los debates de educación y género. Principalmente se ha indagado con documentación sobre la temática utilizando palabras clave como cine y coeducación, cine y género en bases de datos como Dialnet o buscadores como Google Academico (actividad 3). Además, han realizado la visualización de manera autónoma de películas interesándose en quién ejerce la 
dirección, cuáles son los roles asignados y cuáles las representaciones que se hacen tanto en el texto narrado como mostrado sobre las mujeres desde una perspectiva crítica. La lectura de trabajos de investigación ha permitido cuestionar su propio enfoque y adquirir herramientas analíticas desde una perspectiva de género.

Por otro lado, el alumnado ha desarrollado estrategias de investigación social con expertas sobre cine dirigido por mujeres, educación y género (objetivo 2), aprendiendo a realizar el diseño y puesta en marcha de instrumentos de investigación social como la entrevista, la observación y el análisis documental. Este primer acercamiento a la investigación por parte del alumnado les ha conferido, con la orientación y tutela de las profesoras, competencias iniciales para explorar en la elaboración de protocolos de entrevistas, el contacto y la negociación para su realización, desarrollarlas y grabarlas, transcribirlas de forma literal y ensayar el análisis descriptivo de forma iniciática (actividad 4). Del proceso ha quedado constancia en los informes de ApS realizados por el alumnado.

Las profesoras han adoptado un rol de orientadoras-tutoras, guías del proceso de aprendizaje de los y las estudiantes proporcionando tanto los contactos personales para realizar las entrevistas, como formación metodológica específica para el desarrollo de esta técnica de investigación social y del resto de actividades académicas que componen el proceso de ApS.

Con el objetivo de atender al proceso de realización de las entrevistas, se recogieron algunas evidencias informales que permitieron conocer el trabajo del alumnado durante el desarrollo de esta actividad. En concreto, se contactó con las expertas y se les preguntó sobre la experiencia siendo entrevistadas por el alumnado. De este modo, se ha obtenido información relevante que permitió identificar fortalezas y debilidades del propio proceso enseñanza-aprendizaje. Sirva de ejemplo el extracto de la conversación mantenida por parte del profesorado con una de las colaboradas entrevistadas por el alumnado:

(...) La segunda entrevista se concertó el día de la proyección del hilo de la luna, en vivo y en directo (...) Se realizó igualmente en un despacho de la facultad, sin interrupciones y grabada en el móvil. Tampoco yo disponía de guion previo, pero se desarrolló con ambiente relajado y distendido, partiendo de un acercamiento personal a mi trayectoria y procesos de trabajo referidos a los temas de género y cinematografía (...) (Marina Fuentes Guerra, Colaboradora del proyecto)

En otros, se han puesto de manifiesto la necesidad de seguir trabajando en aspectos relacionados con las diferencias entre entrevista y cuestionario o las características y fases fundamentales de la técnica:

(...) por mi parte no me han solicitado entrevista personal, tan sólo el cuestionario que les rellené y que tal vez por ser el último encontré más elaborado (...) Supuse que habían corregido algunos fallos y deficiencias que en los primeros eran más evidentes. Se ve que son muy jóvenes e inexpertas y este es uno más de los muchos trabajos pendientes de entrega. (Araceli Velasco, Colaborada del proyecto).

Con respecto al contenido, se han evidenciado la falta de conocimientos previos, sobre todo desde una perspectiva de género y feminista. Lo cual evidencia la relevancia y novedad del trabajo realizado, el interés surgido y la utilidad de sus resultados:

Pude detectar las lagunas que siguen existiendo en cuanto a la formación en el mundo de la imagen, siendo el mundo más habitual en su cotidianidad. Sobre el cine menos comercial, está prácticamente ausente de sus vidas y de directoras en la inexistencia. 
Creo que es interesante insistir en el aula sobre el potencial didáctico del cine en cuanto a la visualización, reflexión y expresión de todo un mundo emocional, moral, valores, lenguajes, etc. (...) (Araceli Velasco, Colaborada del proyecto).

Las dificultades organizativas, tanto de calendario como de exceso de trabajos que expresa tener el alumnado cuando realiza su trabajo, es también puesto de manifiesto por las expertas que son entrevistadas:

Los dos grupos valoraron el interés de trabajar desde las asignaturas de este modo y se las veía muy interesadas, quizá el problema puede referirse a los tiempos de que disponen y los lugares, con desplazamiento o no, para realizar las entrevistas. Ese horario de clases, apretado y dividido, hace difícil cuadrarlo con tiempos más flexibles y abiertos para prácticas como estas (Marina Fuentes Guerra, Colaboradora del proyecto).

A pesar del poco recorrido en la temática y en el uso de la entrevista como instrumento de investigación por parte del alumnado, el encuentro resultó enriquecedor por ambas partes (Mi valoración global es positiva en cuanto que supone un contacto intergeneracional que nos enriquece mutuamente, Araceli Velasco, Colaborada del proyecto) y permitió ampliar conocimientos, experiencias, saberes y prácticas sobre cine y su reflexión feminista (objetivo 2):

Les proporcioné algunas de las hojas de difusión de las diferentes actividades que hemos realizado sobre cine de mujeres y las animé a visitar algunas páginas web que podían interesarles (Consuelo Borreguero, Colaborada del proyecto).

En definitiva, los testimonios de las expertas coinciden en señalar la oportunidad que el uso del cine tiene como herramienta educativa para el desarrollo de una perspectiva crítica y feminista en el alumnado durante el proceso de enseñanzaaprendizaje:

Creo que es interesante insistir en el aula sobre el potencial didáctico del cine en cuanto a la visualización, reflexión y expresión de todo un mundo emocional, moral, valores, lenguajes, etc. (...) (Araceli Velasco, Colaborada del proyecto).

Espero que les sirva, aunque sólo sea para que no se pregunten si el cine es una herramienta educativa, sino que se cuestionen si el mensaje educativo propuesto es el adecuado" (Consuelo Borreguero, Colaborada del proyecto).

\subsection{Aprendizajes Experienciales}

En relación con el servicio diseñado (objetivo 5) y desarrollado (objetivo 6) por el estudiantado han destacado tres formatos distintos que se corresponden a su vez con los resultados obtenidos tras su exploración bibliográfica y documental: (1) diseño, organización y dirección de un videoforum; (2) elaboración de un vídeo para denunciar sesgos de género en el cine y (3) actividades de concienciación y visibilidad de las mujeres en el cine.

Con respecto al primer servicio, el diseño, organización y dirección de un videoforum, el equipo de trabajo contactó con la productora Tus Ojos ${ }^{5}$ y procedió a hacer una invitación formal para proyectar la película Semillas de Alegría (2019) en la Facultad de Ciencias de la Educación (Figura 5). En este caso se llevó a cabo la actividad en coordinación con la asignatura Educación mediática y su dimensión educativa en el Grado de Educación Infantil lo que permitió conectar contenidos relacionados con el uso de los medios audiovisuales desde una perspectiva educativa.

\footnotetext{
${ }^{5}$ Agradecemos la asistencia de Manuel Serrano miembro de la productora Tus Ojos.
} 

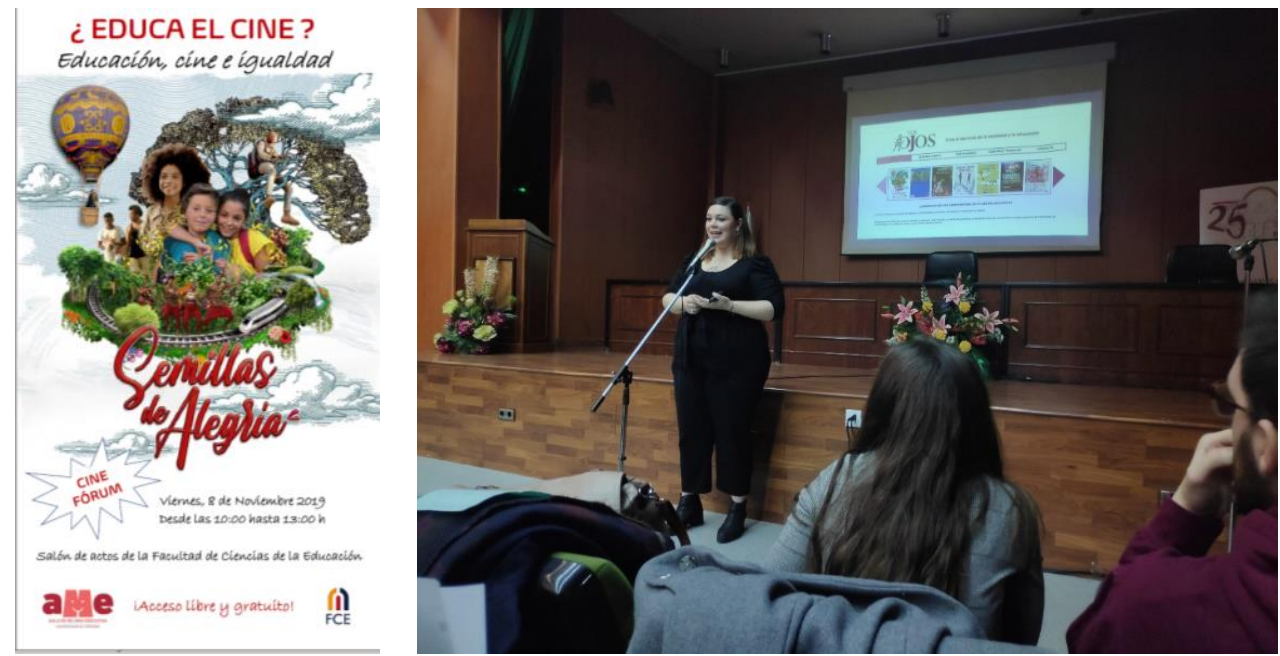

Figura 5. Servicio de grupo de estudiantes: proyección de la película Semillas de Alegría (2019). Cartel y presentación del videoforum.

Dicha actividad ha reportado aprendizajes que basados en la experiencia ha motivado la indagación y la reflexión. El alumnado lo expresaba de la siguiente manera en el trabajo escrito:

El descubrimiento de Cristina Linares y su obra, se llevó a cabo a través de la organización Tus Ojos, cine al servicio de la sociedad y la educación. Esta entidad se encarga de seleccionar, crear y promover contenidos audiovisuales que cumplan unos objetivos de transmisión de: conductas positivas, fomento de la solidaridad, igualdad de género y protección de la infancia. Además, este colectivo, colabora con instituciones públicas y privadas en proyectos sin ánimo de lucro. Desarrollar actividades en festivales, televisión, internet, aulas, universidades...Pero, sobre todo, también organiza exposiciones itinerantes allí donde pueda haber una pantalla y un equipo para reproducir adecuadamente y, es aquí donde se va a desarrollar nuestro servicio. Para más información sobre Tus Ojos. (Extracto del trabajo escrito del alumnado, 12).

El segundo de los formatos que adoptó el servicio diseñado por los estudiantes consistió en la elaboración de un vídeo con el objetivo de denunciar los sesgos de género en el cine. A pesar de disponer como único recurso para su elaboración de los medios audiovisuales de los estudiantes, los datos que ofrece han tenido una buena acogida en la plataforma digital depositada ${ }^{6}$.

Otros servicios han consistido en el diseño y realización de actividades para la toma de conciencia de la invisibilidad de las mujeres dirigiendo cine y de los sesgos patriarcales que el cine transmite en sus contenidos. Estas actividades han sido expuestas de manera pública y lúdica en las zonas comunes de la Facultad en el evento Feria de la Convivencia al finalizar la asignatura (Figura 6).

\footnotetext{
${ }^{6}$ Disponible en: https://www.youtube.com/watch?v=N90xH005rhA\&feature=youtu.be
} 

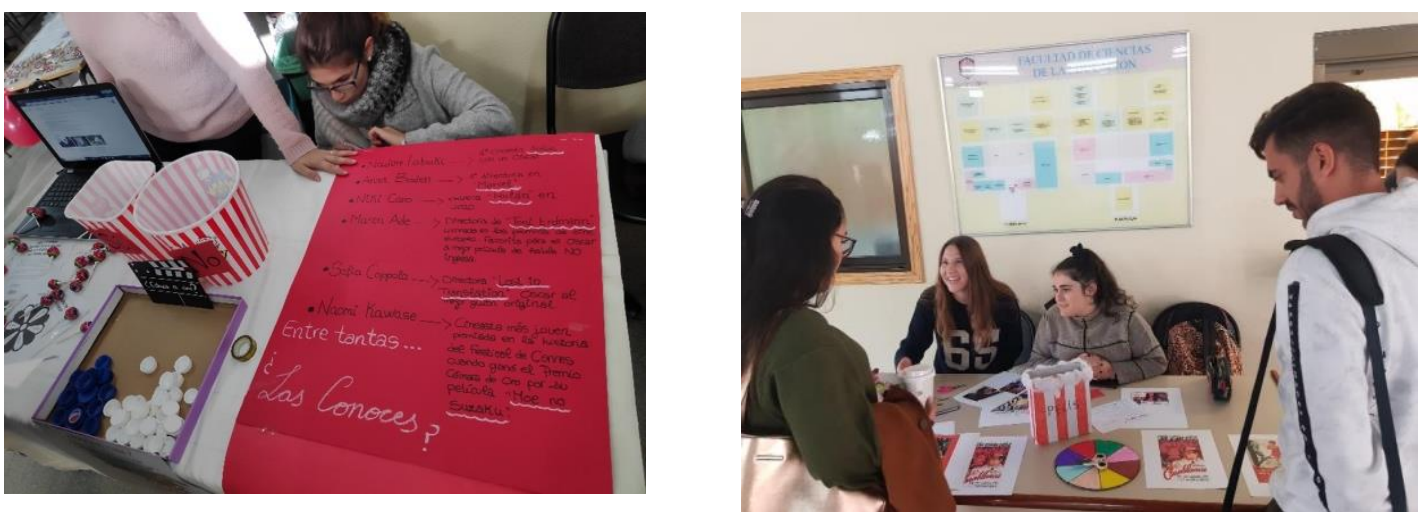

Figura 6. Participación en Feria de Convivencia, grupos Grado de Educación Infantil y Primaria

Además del diseño y desarrollo del servicio, los aprendizajes experienciales han sido complementados con la búsqueda y visualización de manera autónoma del estudiantado de películas dirigidas por mujeres durante el proceso de aprendizaje (objetivo 3); pero principalmente, de manera más dirigida, mediante la actividad organizada por el profesorado consistente en el visionado y debate del documental Irioweniasi. El hilo de la luna (2018). A la sesión fueron invitadas sus directoras ${ }^{7}$ lo que permitió el diálogo y reflexión sobre el contenido abordado en el trabajo audiovisual (Figura 7). Este trabajo estimuló la indagación y análisis de otros trabajos y películas dirigidas por mujeres y experiencias concretas para el uso educativo del cine (objetivo 4), pero sobre todo fue una actividad que despertó su interés vinculando al trabajo desde las escuelas. Varias estudiantes lo manifestaban del siguiente modo en el cuestionario elaborado para valorar la actividad:

Me ha parecido muy interesante, muchas personas deberían ser conscientes de cosas como las de esta charla. (Cuestionario valoración actividad, Mujer, I2).

Maravillosa actividad, ojalá fomentar más actividades de este tipo que nos hacen parar y reflexionar. (Cuestionario valoración actividad, Mujer, I1).

Ha sido verdaderamente interesante y muy enriquecedor (Cuestionario valoración actividad, Mujer, I3).
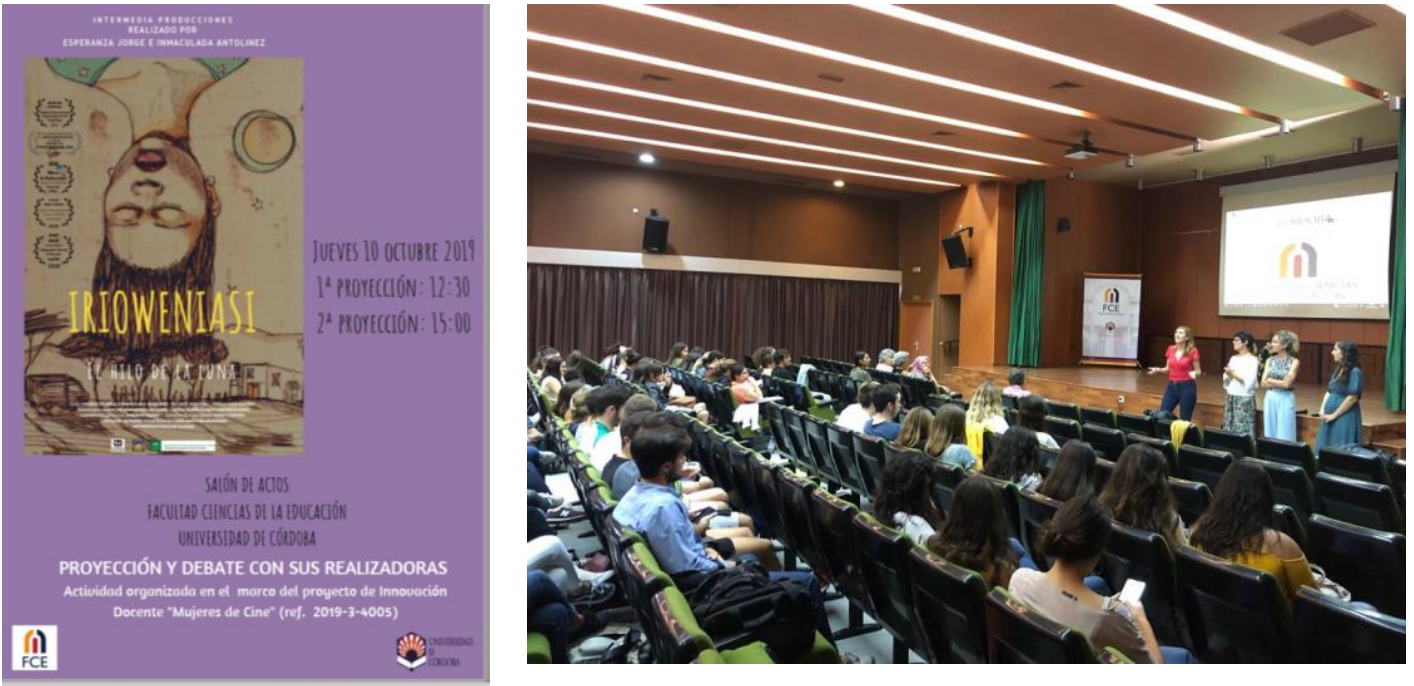

Figura 7. Cartel y momento de la sesión en la que se proyectó el documental.

\footnotetext{
7 El equipo de profesorado del proyecto agradece la participación de Esperanza Jorge e Inmaculada Antolínez y la proyección del documental que han dirigido y la posterior sesión de trabajo mantenida con el alumnado y por duplicado en la Facultad de Ciencias de la Educación.
} 


\subsection{Conexión teórico $\leftrightarrow$ experiencial para construir una ciudadanía crítica}

La última parte del proceso metodológico consistió en la producción de un informe por parte del alumnado dando cuenta de todo el proceso seguido tanto metodológico como conceptual. Además, resultó especialmente relevante la puesta en valor de las capacidades del alumnado para establecer conexiones entre la indagación teórica realizada y el servicio diseñado e implementado, en su caso (objetivo 7).

Varios son los aportes de esta metodología. Por un lado, la autonomía y la conexión teórico-práctica que se les requiere permite alejarnos de un aprendizaje memorístico, todavía presente en la formación inicial del profesorado, para dar paso a un aprendizaje competencial, vivencial y reflexivo. Esto es reconocido y valorado por el estudiantado como pone de manifiesto una estudiante en su informe: Me aburro fácilmente si siempre aprendo solo con apuntes prefijados, con todo medido y estructurado, la verdad, realizar trabajos de este tipo me dan una motivación que me hace mucha falta (estudiante GEI). Por otro lado, situar en el centro al discente y darle la oportunidad de elección libre de la temática y el diseño del servicio, favorece su inicial motivación y elección en equipo de forma más acorde a sus intereses; sin embargo, al mismo tiempo, puede crearles inseguridades iniciales que le suponen dificultades en el cumplimiento de la tarea académica:

En un primer momento, me costó bastante entender el concepto de la dinámica del trabajo, es decir, la metodología y cómo abordarlo y ponerlo en práctica, cuando la profesora lo explicó me invadieron muchas sensaciones, por un lado, había algo que me gustaba, por otro, me asustaba y lo veía muy extenso, muy etéreo (estudiante GEI).

No obstante, el trabajo autónomo del grupo, presencial y no presencial, supone un proceso de aprendizaje sobre la autogestión del tiempo y la organización de éste con potencialidad para su propia formación. Además, la aplicación de los conocimientos teóricos para la realización del servicio ha posibilitado que el alumnado trabajara con motivación y creatividad, reflejándose en la diversidad de trabajos y formatos propuestos. Por tanto, conectar la teoría con la puesta en marcha de un servicio, les ha permitido contar con herramientas de análisis teóricas y contextuales oportunas para todo un proceso que comienza con identificación de una problemática, su definición, la indagación, el diseño de una acción, su implementación, la difusión y evaluación:

Nos ha brindado la oportunidad para estudiar e informar al resto de la gente uno de los temas que más importancia debe tener ya que une dos ámbitos característicos hoy en día como lo son la desigualdad de la mujer respecto al hombre y el mundo del cine, siendo uno de los recursos más utilizados por todas las edades para transmitir conocimientos, emociones y actitudes (estudiante, GEP).

Por otro lado, el trabajo en equipo sigue siendo una de las debilidades que el alumnado manifiesta en la realización de su trabajo. Así queda expresado en los informes escritos al reconocer las dificultades para realizar un trabajo desde presupuestos cooperativos y colaborativos: aunque me gustaría recalcar la participación y ayuda de solo una de las cuatro integrantes del grupo. Solo dos nos hemos empapado y rodeado de más aprendizajes (estudiante, GEI).

Asimismo, algunos aspectos no resueltos y que se vuelven a mostrar como debilidad, como en otras asignaturas y momentos del aprendizaje del alumnado universitario, las dificultades tenidas en la elaboración de tareas académicas vinculadas con la competencia de escritura académica y la aún incipiente formación en 
propedéutica en este nivel de grado universitario. La realización de actividades formativas transversales en el grado podría servir para potenciar, reforzar y mejorar esta competencia en el alumnado.

Estos resultados son coincidentes con estudios previos sobre ApS (Andrea y Moliner, 2010) al destacar que trabajar en equipos pone a prueba las oportunidades que surgen para practicar habilidades sociales y cooperativas como son el diálogo, la escucha activa, el consenso, la negociación, asertividad, la empatía, el respecto, la solidaridad o el compromiso. Experiencias docentes que han empleado el cine como herramienta educativa y cuestionan la desigualdad en relación con el género (Alfaya, Bueno y Navarro, 2012) también coinciden en ese mismo sentido. La novedad de aunar los aportes del ApS y el cine en clave de género en el mismo proceso ha servido en este sentido para el trabajo pretendido vinculado al trabajo por competencias académicas, sociales, ciudadanas y críticas.

\section{CONCLUSIONES}

Este proyecto de innovación docente ha permitido abrir las puertas del aula a través del mensaje fílmico para promover la ciudadanía crítica y responsable, como competencia ineludible en la formación inicial del profesorado. Trabajamos con los imaginarios colectivos y con estrategias de indagación y construcción de recursos que permitan narrar las imágenes y los textos en clave de justicia y equidad. El masivo consumo del lenguaje audiovisual hace especialmente interesante y relevante este propósito.

En este proyecto se han evidenciado fortalezas que afianzan la apuesta planteada al comienzo. Entre ellas, el hecho de contar con colaboradoras expertas en la temática de cine y coeducación con disponibilidad para que el alumnado interaccionara con ellas a través de la realización de entrevistas ha sido un recurso humano fundamental para el trabajo del alumnado, su aprendizaje vivencial a través de la investigación. Además, la presencia de trabajos audiovisuales en el aula y de películas con interés social, no comercial ha permitido al alumnado poner rostro a proyectos educativos, profundizar en el mensaje del material fílmico y contar con experiencias de aprendizaje que conectaran el contenido con el contexto social.

Las potencialidades de la metodología de ApS favorecen la indagación, reflexión y acción y las acciones desarrolladas lo han posibilitado.

El proyecto de innovación docente ha permitido el trabajo en equipo del profesorado que trabaja en los tres grupos del Grado de Educación Infantil y en coordinación con el Grado de Educación Primaria, analizando y reflexionando de forma conjunta sobre la práctica docente universitaria. Asimismo, la participación del personal experto junto al equipo docente genera un espacio interdisciplinar que ha enriquecido el conocimiento. Esta es una oportunidad para la creación de redes de colaboración intra y extraacadémicas a favor de procesos de enseñanza-aprendizaje vinculados a experiencias sociales no ficticias.

A pesar de que los puntos fuertes han superado las debilidades, éstas se relacionan aspectos como: el trabajo monográfico sobre cine y coeducación sólo es realizado por los grupos que lo seleccionan; o el hecho de contar con una débil participación por parte del alumnado en actividades de carácter cultural vinculadas a la asignatura si no obtienen a cambio compensación en su calificación; desconocimiento por parte del estudiantado sobre cine y teoría de género lo que permite solo un acercamiento inicial y un análisis superficial de la temática; y la existencia de dificultades organizativas para realizar actividades fuera de los márgenes temporales o espaciales institucionales.

Si bien este proyecto no contempla realizarse en sucesivos años, puede servir para ser transferido a otras asignaturas y contextos. Algunos elementos que podrían servir para mejorar el trabajo realizado son: ampliar la coordinación con el profesorado de otras asignaturas del curso para la realización de un proyecto global, acorde a las competencias y contenidos impartidos en cada una de ellas; dotar de apoyo técnico que 
sirva de soporte para la realización de material audiovisual por parte del alumnado cuando así lo planifique como parte del servicio a la comunidad, de esta manera se podría contar con trabajos de mayor calidad audiovisual que repercutiría, a su vez, en mayor aprendizaje, posibilidades de difusión y su evaluación e impacto.

Aunar ApS, cine y coeducación ha multiplicado las posibilidades de trabajar a favor de la transformación social desde la Pedagogía crítica promoviéndose el desarrollo de una de las funciones fundamentales de la Universidad: formar una ciudadanía crítica, activa y responsable con la sociedad actual.

\section{REFERENCIAS}

Alfaya Góngora, M. M., Bueno M. J., \& Navarro, S. (2012). Estereotipos y roles: mujeres en el cine. In I Congreso Internacional de Comunicación y Género. Libro de Actas: 5, 6 y 7 de marzo de 2012. Facultad de Comunicación. Universidad de Sevilla. (pp. 1146-1157). Facultad de Comunicación. Recuperado de https://dialnet.unirioja.es/servlet/articulo?codigo $=5383300$

Aguilar, P. (2017). El papel de las mujeres en el cine. Madrid: Santillana

Almacellas, M.A. (2019). El cine, una escuela de sabiduría o cómo convertir cada película en una lección de ética. Cuadernos de Pedagogía, 499, 218-221.

Bonilla, J. (2008). Educación en valores a través del cine. Un método para estudiantes de secundaria. Tesis doctoral, Departamento de Psicología Social, Universidad de Sevilla.

Chiva, Ò., Gil, J., Corbatón, R. y Capella, C. (2016). El aprendizaje servicio como propuesta metodológica para una pedagogía crítica. RIDAS, Revista Iberoamericana de Aprendizaje Servicio, 2, 70-94.

Cruzado, M.A. (2009). Mujeres y Cine. Discurso Patriarcal y Discurso Feminista, de los textos a las pantallas (Tesis Doctoral). Facultad Filología, Universidad de Sevilla.

Deeley, S. J. (2016). El aprendizaje-servicio en educación superior. Teoría, práctica y perspectiva crítica. Madrid: Narcea.

Fueyo Gutiérrez, A. y Fernández del Castro, J.I. (2012). Hacer visible lo cotidiano a través del cine: la perspectiva de género en la Educación para el Desarrollo. REIFOP, 15 (2).

González Jiménez, D. A. (2012). Re-encuadrar dentro del encuadre: miradas a un encuentro cine-educación. Revista Colombiana de Educación, (63),131-152. Recuperado de https://www.redalyc.org/articulo.oa?id=4136/413635256011

González Párraga, E. (2019). El cine como herramienta en el aula: una guía práctica. Cuadernos de Pedagogía, 501, 122-125.

Heredero de Pedro, Carmen (2019). Género y coeducación. Madrid: Morata.

Jorge, E.; Antolínez, I. y Cordero, N. (2017). Iriowenias, el hilo de la luna. Sevilla, Benilde Ediciones.

Margalef, L. y Canabal, C. (Dirs.) (2013). Innovar en la enseñanza universitaria. Madrid: Biblioteca Nueva.

Martínez-Salanova, E. (2003). El valor del cine para aprender y enseñar. Comunicar: Revista Científica de Comunicación y Educación, 10(20), 45-52.

Morales, B. (2015). Roles y estereotipos de género en el cine romántico de la última década. Perspectivas educativas. Tesis doctoral, Universidad de Sevilla. Recuperado de https://gredos.usal.es/jspui/bitstream/10366/128238/1/DTHE_MoralesRomoB_ Rolesg\%C3\%A9nerocine.pdf

Naranjo, M. (2020). Del desarrollo individual a la transformación social: Potencialidades de los modelos ApS para transformar las necesidades y retos de la juventud 
española. En E. J. Díez y J. R. Rodríguez Fernández (Dirs) Educación para el Bien Común. Hacia una práctica crítica, inclusiva y comprometida socialmente. Barcelona: Octaedro. Recuperado de https://octaedro.com/wpcontent/uploads/2020/02/16215-Educacion-para-el-Bien-Comun.pdf

Núñez Domínguez, T. (2010). Mujeres directoras de cine: un reto, una esperanza. PixelBit, 37, 121-133.

Perales, F. (2011). La mujer como imagen de marca en el cartel de cine español en el periodo 2010-2013. Historia y Comunicación Social, Especial, 189-201.

Puig, J. M. y Palos, J. (2006). Rasgos pedagógicos del aprendizaje-servicio. Cuadernos de Pedagogía, 357, 60-63. Recuperado de https://dialnet.unirioja.es/servlet/articulo?codigo=1970468

Soler García, C; Quintanilla Batallanos, V. A. y Aguilar Trujillo, D. (2018). La Formación Inicial del Profesorado. Un Proceso Democrático. Revista Interuniversitaria de Formación del Profesorado. 92 (32.2), 107-122. Recuperado de https://dialnet.unirioja.es/servlet/articulo?codigo $=6627821$

Torres, J. (2017). Políticas educativas y construcción de personalidades neoliberales y neocolonialistas. Madrid: Morata.

Zeichner, K. M. y Flessner, R. (2010). Educar al profesor para la justicia social. En K. M. Zeichnner La formación del profesorado y la lucha por la justicia social. Madrid: Morata. 